\title{
SUSCEPTIBILITY PATTERNS AND CLINICAL MANAGEMENT OF HOSPITALISED CHILDREN WITH URINARY TRACT INFECTIONS IN CENTRAL GREECE
}

1,2,3Vazouras K, ${ }^{3}$ Velali K, ${ }^{4}$ Tassiou I, ${ }^{3}$ Anastasiou-Katsiardani A, ${ }^{3}$ Athanasopoulou K, ${ }^{5}$ Barbouni A, ${ }^{2}$ Folgori L, ${ }^{2,6}$ Zaoutis T, ${ }^{7}$ Basmaci R , ${ }^{2} \mathrm{Hsia} Y$

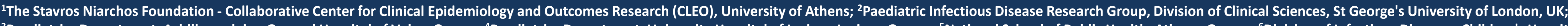

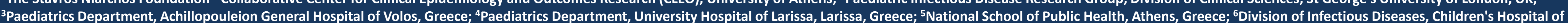

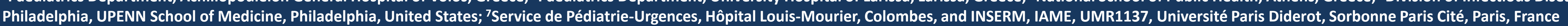

BACKGROUND

- There is limited evidence on paediatric UTIs prescribing in Greece The high antimicrobial resistance (AMR) rates and inappropriate antibiotic prescribing are posing an increasing threat to public health in Greece

AIMS

Retrospective cohort study aiming to:

- describe antibiotic prescribing patterns

evaluate resistant rates in hospitalised children with febrile and afebrile UTIs in a district hospital in central Greece

Table 1: Demographics

\begin{tabular}{|c|c|c|c|c|}
\hline Demographics & $\begin{array}{l}\text { All UTIs } \\
(\mathrm{n}=230)\end{array}$ & $\begin{array}{l}\text { Febrile UTIs } \\
(\mathrm{n}=181)\end{array}$ & $\begin{array}{l}\text { Afebrile } \\
\text { UTIs ( } n=49)\end{array}$ & $\begin{array}{l}p- \\
\text { value }\end{array}$ \\
\hline \multicolumn{5}{|l|}{ Age (on admission) } \\
\hline$<29$ days & $15(6.5 \%)$ & $12(6.6 \%)$ & $3(6.1 \%)$ & \multirow{4}{*}{0.07} \\
\hline 1 month $-<2$ years & $138(60.0 \%)$ & 116 (64.1\%) & $22(44.9 \%)$ & \\
\hline 2 years $-<5$ years & $37(16.1 \%)$ & $25(13.8 \%)$ & $12(24.5 \%)$ & \\
\hline$\geq 5$ years & $40(17.4 \%)$ & $28(15.5 \%)$ & $12(24.5 \%)$ & \\
\hline \multicolumn{5}{|l|}{ Sex } \\
\hline Male & $64(27.8 \%)$ & $49(27.1 \%)$ & 15 (30.6\%) & \multirow[b]{2}{*}{0.72} \\
\hline Female & $166(72.2 \%)$ & $132(72.9 \%)$ & $34(69.4 \%)$ & \\
\hline \multicolumn{5}{|l|}{ Background } \\
\hline Previously healthy children & $181(78.7 \%)$ & $141(77.9 \%)$ & $40(81.6 \%)$ & 0.70 \\
\hline UT-abnormalities ${ }^{a}$ & $25(10.9 \%)$ & $24(13.3 \%)$ & $2(0.04 \%)$ & 0.08 \\
\hline Other medical conditions ${ }^{b}$ & $24(10.4 \%)$ & $17(9.4 \%)$ & $7(0.14 \%)$ & 0.30 \\
\hline Concurrent infections & $11(4.8 \%)$ & $11(6.1 \%)$ & $0(0.0 \%)$ & 0.13 \\
\hline Bacteraemic UTls & $6(2.6 \%)$ & $6(3.3 \%)$ & $0(0.0 \%)$ & 0.35 \\
\hline Recurrent UTIs & $40(17.4 \%)$ & $35(19.3 \%)$ & $5(0.1 \%)$ & 0.20 \\
\hline Atypical UTIs & $96(41.7 \%)$ & $78(43.1 \%)$ & $18(36.7 \%)$ & 0.74 \\
\hline Recent hospitalisation & $10(4.3 \%)$ & $8(4.4 \%)$ & $2(0.04 \%)$ & 1 \\
\hline $\begin{array}{c}\text { Recent/concurrent antibiotics use } \\
\text { Pathogens }\end{array}$ & $29(12.6 \%)$ & $27(14.9 \%)$ & $2(0.04 \%)$ & 0.05 \\
\hline E. coli & $187(79.2 \%)$ & $153(81.8 \%)$ & $34(69.4 \%)$ & 0.07 \\
\hline Klebsiella sp & $17(7.2 \%)$ & $14(7.5 \%)$ & $3(6.1 \%)$ & 1.00 \\
\hline Proteus sp & $12(5.1 \%)$ & $8(4.3 \%)$ & $6(12.2 \%)$ & 0.05 \\
\hline Pseudomonas aeruginosa & $11(4.7 \%)$ & $6(3.2 \%)$ & $3(6.1 \%)$ & 0.40 \\
\hline Enterobacter sp & $4(1.7 \%)$ & $2(1.1 \%)$ & $2(4.1 \%)$ & 0.19 \\
\hline Citrobacter sp & $1(0.4 \%)$ & $1(0.5 \%)$ & $0(0.0 \%)$ & 1 \\
\hline Gram $(+)$ coccic & $4(1.7 \%)$ & $3(1.6 \%)$ & $1(2.1 \%)$ & 1 \\
\hline Total & $236(100 \%)$ & $187(100 \%)$ & $49(100 \%)$ & \\
\hline
\end{tabular}

METHODS

- Study period: August 2010 to September 2016

- Included children 0-17 years old

- Patients records retrieved using ICD-10 codes

- Data was collected on antibiograms and antibiotic prescriptions

- Patients were included based on clinical and microbiological criteria

- Sensitivity to antimicrobials was determined using the Kirby-Bauer disk diffusion method

Table 2. Antibiotic prescriptions for febrile and afebrile UTIs treatment

\begin{tabular}{|c|c|c|c|c|c|c|}
\hline Antibiotic & $\begin{array}{l}\text { No of hospital } \\
\text { prescriptions }\end{array}$ & $<29$ days & $1 m-2 y$ & $2 y-5 y$ & $5 y-18 y$ & $\begin{array}{l}\text { Treatment duration in } \\
\text { days (Mean; SD) }\end{array}$ \\
\hline fUTIs & $n=378$ & & & & & \\
\hline Amikacin & 124 (32.8\%) & 10 & 82 & 14 & 18 & $(4.4 ; 1.9)$ \\
\hline AMC & $63(16.7 \%)$ & 1 & 45 & 10 & 7 & $(5.8 ; 2.1)$ \\
\hline Ampicillin & $53(14.0 \%)$ & 10 & 37 & 4 & 2 & $(3.9 ; 2.7)$ \\
\hline Cefotaxime & 42 (11.1\%) & 8 & 25 & 5 & 4 & $(6.7 ; 2.7)$ \\
\hline SAM & 34 (9.0\%) & & 21 & 4 & 9 & $(4.8 ; 1.7)$ \\
\hline Cefuroxime & 25 (6.6\%) & & 14 & 3 & 8 & $(4.3 ; 1.9)$ \\
\hline Ceftriaxone & $17(4.5 \%)$ & & 10 & 4 & 3 & $(8.4 ; 2.2)$ \\
\hline TAZ & $7(1.9 \%)$ & 1 & 5 & & 1 & $(7.4 ; 4)$ \\
\hline Ceftazidime & $3(0.8 \%)$ & & 2 & & 1 & 6.7 \\
\hline Cefoxitin & $2(0.5 \%)$ & & 2 & & & 3.7 \\
\hline TMP/SMZ & $2(0.5 \%)$ & & 2 & & & - \\
\hline Other & $6(1.8 \%)$ & 1 & 4 & & 1 & - \\
\hline aUTIs & $\mathrm{n}=81$ & & & & & \\
\hline Amikacin & $19(23.5 \%)$ & 3 & 14 & 1 & 1 & $(5 ; 2.5)$ \\
\hline AMC & $17(21.0 \%)$ & 1 & 12 & 1 & 3 & $(4 ; 2.3)$ \\
\hline Cefuroxime & $13(16.0 \%)$ & & 7 & 3 & 3 & $(3.6 ; 1.6)$ \\
\hline Ampicillin & 9 (11.1\%) & 2 & 6 & & 1 & $(4.9 ; 4)$ \\
\hline SAM & $5(6.2 \%)$ & & 2 & 2 & 1 & $(3.4 ; 1.5)$ \\
\hline Cefprozil & $4(4.9 \%)$ & & & 2 & 2 & $(1.3 ; 0.3)$ \\
\hline TAZ & $3(3.7 \%)$ & & 2 & & 1 & $(7.7 ; 0.3)$ \\
\hline Cefaclor & $3(3.7 \%)$ & & & 2 & 1 & \\
\hline Cefotaxime & $3(3.7 \%)$ & 1 & 2 & & & $(7.7 ; 2.5)$ \\
\hline Other & 5 (6.1\%) & & 3 & 1 & 1 & \\
\hline
\end{tabular}

Abbreviations: SAM, ampicillin-sulbactam; AMC, amoxicillin-clavulanic acid; TLV, ticarcillinclavulanic acid; TAZ, piperacillin-tazobactam; TMP/SMZ, trimethoprim-sulfamethoxazole
RESULTS

- Two hundred thirty patients were included

Among 459 prescriptions identified, amikacin (31.2\%) was the most commonly antibiotic prescribed in this population (Table 2)

- The following most common antibiotics were amoxicillinclavulanic acid (17.4\%) and ampicillin (13.5\%) (Table 2)

- Children received prolonged intravenous treatments for febrile (5.4 days; SD 1.45) and afebrile UTIs (4.7 days; SD 1.34)

- Two-hundred thirty-six pathogens isolated (Table 1)

- The main causative organism was Escherichia coli (79.2\%) with high reported resistance rates to ampicillin (42.0\%) trimethoprim-sulfamethoxazole $(26.5 \%)$ and amoxicillin/clavulanic acid (12.2\%)

- Lower resistance rates of $E$. coli to 3rd generation cephalosporins (1.7\%), nitrofurantoin (2.3\%), ciprofloxacin $(1.3 \%)$ and amikacin $(0.9 \%)$

- Klebsiella spp. isolates were highly resistant to cefaclor (27.3\%)

CONCLUSION

UTIs is a key area to monitor antimicrobial resistance in childhood

- The establishment of antimicrobial stewardship programs could help to minimise inappropriate prescribing for UTIs in Greek hospitals

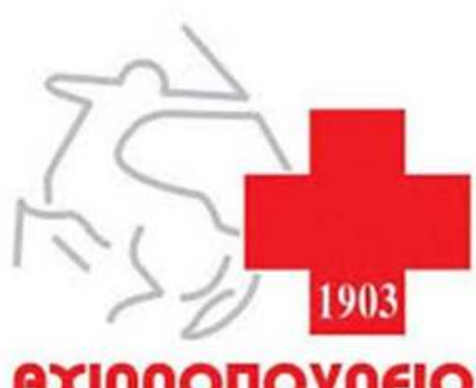

REFERENCES 1. Bryce A, BMJ 2016; 352::939. Miyakis S, Clin Infect Dis 2011; 53(2):177-84. 3. Kourlaba G, J Antimicrob Chemother 2015 70(8):2405-8 